\title{
Paralysie faciale périphérique et soins dentaires : lien ou coïncidence, pour quelle prise en charge?
}

\author{
Romy Makhoul ${ }^{1}$, Jennifer Even ${ }^{1}$, Yannick Sudrat ${ }^{1,2}$, Laurent Devoize ${ }^{1,2,3,{ }^{*}}$, Cédric Huard ${ }^{1,2}$ \\ 1 Service d'Odontologie, CHU, Clermont-Ferrand, France \\ 2 Faculté de Chirurgie dentaire, Université d'Auvergne, Clermont-Ferrand, France \\ 3 UMR Inserm/UdA, U1107, Neuro-Dol, Trigeminal Pain and Migraine, Faculté de Chirurgie dentaire, Clermont-Ferrand, France
}

(Reçu le 14 septembre 2013, accepté le 15 décembre 2013)

Mots clés : paralysie faciale périphérique / soins dentaires / virus varicelle-zona / traitement

\begin{abstract}
Résumé - Introduction : L'atteinte du nerf facial est angoissante pour le patient du fait de la déformation faciale qu'elle entraîne et pour le praticien qui se sent impuissant. La survenue d'une paralysie faciale périphérique, suite à des soins dentaires, est rare. Lorsqu'elle survient, aucune notion de traumatisme ou d'étiologie iatrogène ne peut être mise en cause de manière formelle. Observation : Le cas d'une patiente atteinte d'une paralysie faciale périphérique de grade IV selon la classification de House-Brackmann apparue suite à une anesthésie loco-régionale au foramen mandibulaire est décrit. Discussion : La notion de réactivation du virus varicelle-zona suite à un stress quel qu'il soit serait l'origine la plus probable. L'American Association of Neurology recommande la mise en place d'un traitement combiné de prednisolone $(1 \mathrm{mg} / \mathrm{kg} / \mathrm{j})$ et d'aciclovir $(400 \mathrm{mg} 5 \times / \mathrm{j})$ pendant 10 jours; il est également admis que toute paralysie faciale doit être prise en charge le plus précocement possible, idéalement dans les 72 heures, le succès du traitement dépendant en grande partie de sa rapidité d'instauration.
\end{abstract}

Key words:

Abstract - Introduction: Any facial nerve injury is a distressing condition for both the patient because of the peripheric facial palsy / dental care / varicella zoster virus / treatment facial deformity it leads and the practitioner who feels powerless. The occurrence of facial palsy following dental care is rare. When it occurs, any notion of trauma or iatrogenic etiology cannot be questioned formally. Observation: This article focuses on the case of a patient with a grade IV peripheral facial palsy according to House-Brackmann's classification, appeared after regional anesthesia at the mandibular foramen. Discussion: The concept of reactivation of varicella-zoster virus following stress would be the most likely origin. The American Association of Neurology recommends the establishment of a combined treatment of prednisolone $(1 \mathrm{mg} / \mathrm{kg} / \mathrm{day})$ and aciclovir ( $400 \mathrm{mg} 5$ times/day) during 10 days, it is also recognized that any facial paralysis should be supported as early as possible, ideally within 72 hours, the success largely depends on the rapidity of introduction of treatment.

\section{Introduction}

Aussi bien appelée «paralysie de Bell » ou « paralysie à frigore », la paralysie faciale est le plus souvent aiguë et d'origine incertaine. On décrit deux types de paralysie faciale : l'une dite centrale, correspondant à une lésion en amont du noyau somato-moteur du VII ; l'autre dite périphérique, résultant d'une lésion de la racine nerveuse du nerf facial siégeant en un point quelconque de son trajet intra- ou extra-crânien [1]. Cet article s'intéresse au cas d'une patiente atteinte d'une paralysie faciale périphérique de grade IV selon la classification de House-Brackmann, apparue après une anesthésie loco-régionale au foramen mandibulaire.

\section{Observation}

Une femme caucasienne, âgée de 46 ans, s'est présentée en consultation pour un bilan bucco-dentaire. L'examen général et l'interrogatoire de la patiente révèlent des antécédents d'éthylisme, de dépression et d'hypothyroïdie. Elle fume également un paquet de cigarettes par semaine depuis une vingtaine d'années. Elle est actuellement sevrée de son intoxication alcoolique et traitée par oxazépam et lévothyroxine sodique.

Un plan de traitement a été établi, le début des soins a été entrepris avec une première séance d'assainissement et de conseils d'hygiène bucco-dentaire. Celle-ci a été suivie de la

\footnotetext{
* Correspondance : laurent.devoize@udamail.fr
} 


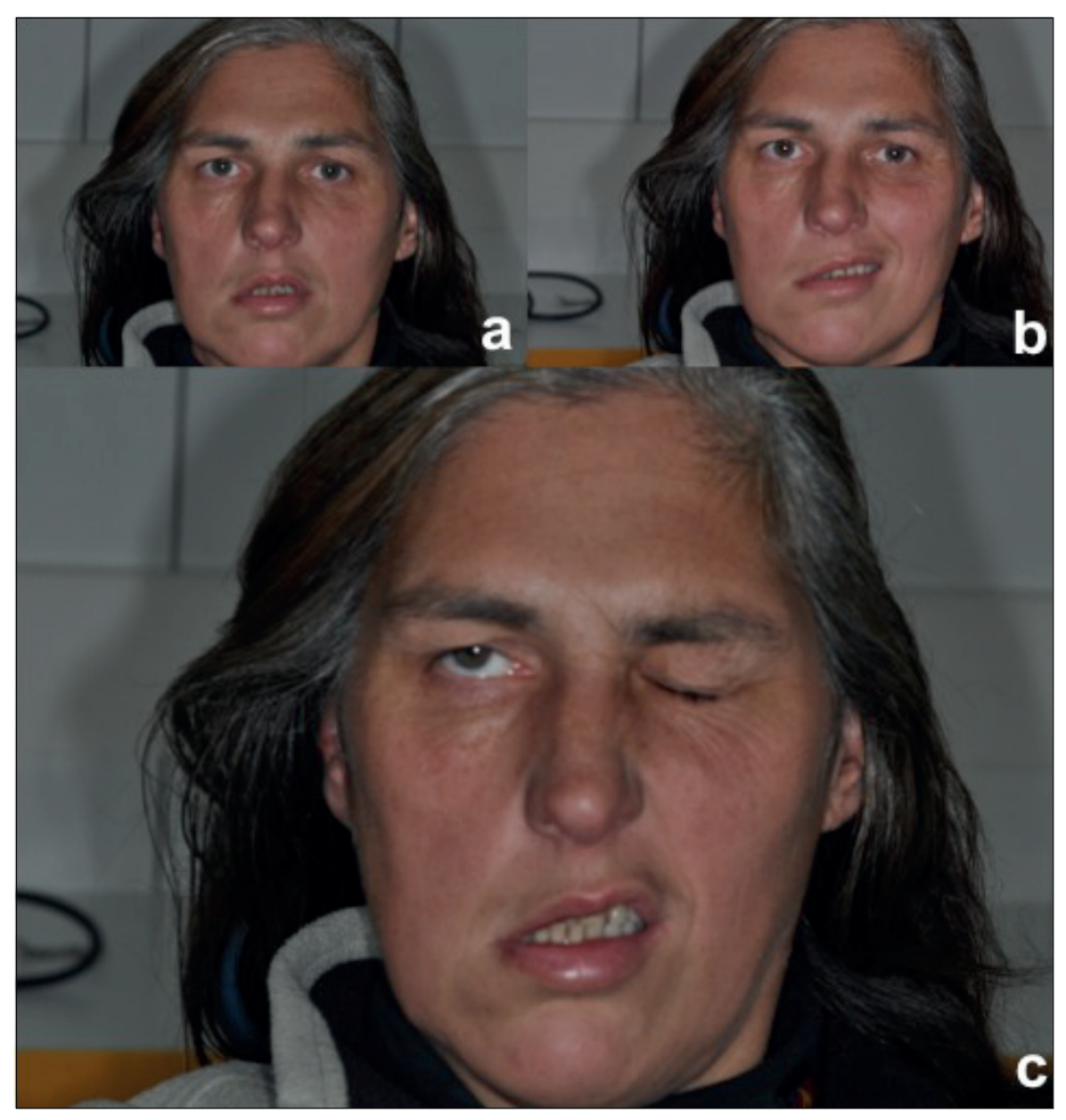

Fig. 1. Évaluation de la paralysie faciale périphérique droite. $a$ : patiente au repos sans mimique faciale ; $b$ : patiente souriante, la lèvre droite est immobile ; $c$ : patiente en occlusion palpébrale, il y a une incompétence totale palpébrale droite et l'œil se porte en haut et en dehors (signe de Charles Bell).

Fig. 1. Evaluation of the right facial peripheric palsy. a: patient at rest without facial expression; $b$ : patient smiling, right lip is stationary; c: patient in eyelid occlusion, there is an absolute lack of eyelids of the right eye with eye eversion (Charles Bell's sign).

réhabilitation prothétique de la seconde prémolaire mandibulaire droite délabrée, pour laquelle il a été décidé de réaliser une restauration indirecte type " onlay ».

Le jour de la pose de la prothèse, une anesthésie locorégionale au foramen mandibulaire a été réalisée en injectant une cartouche d'une solution anesthésique d'articaïne adrénalinée à 1/200000.

La patiente est revenue seulement 6 j plus tard bien qu'elle présentait une perte évidente de la motricité de l'hémiface droite, correspondant à un grade IV selon House-Brackmann (annexe 1). Selon l'interrogatoire, la paralysie s'est installée dans les $24 \mathrm{~h}$ qui ont suivi les soins. La patiente n'a pas consulté en urgence, pensant que cette paralysie était due à la procédure anesthésique.

Au repos, du côté atteint, on observe une ptose génienne basse, un effacement des rides frontales, un élargissement de la fente palpébrale due à l'éversion de la paupière inférieure, un effacement du sillon naso-génien avec attraction de la commissure labiale vers le côté sain et un érythème dans la zone pré-auriculaire droite (zone de Ramsay-Hunt). La patiente rapporte également l'existence d'otalgies depuis quelques jours. Pour les mouvements volontaires, la patiente était dans l'impossibilité de réaliser les mimiques suivantes: siffler, souffler et gonfler la joue du côté atteint. Il existait également un déficit de la fermeture palpébrale droite avec rotation de l'œil en haut et en dehors (signe de Charles Bell) (Fig. 1a, b et c) ainsi qu'un trouble de l'élocution et de l'alimentation.

Le tableau clinique - apparition soudaine d'une paralysie faciale associée à un syndrome de Ramsay-Hunt - a fait évoquer une réactivation du virus varicelle-zona $(V Z V)$. Après interrogatoire, la patiente a confirmé avoir contracté la 


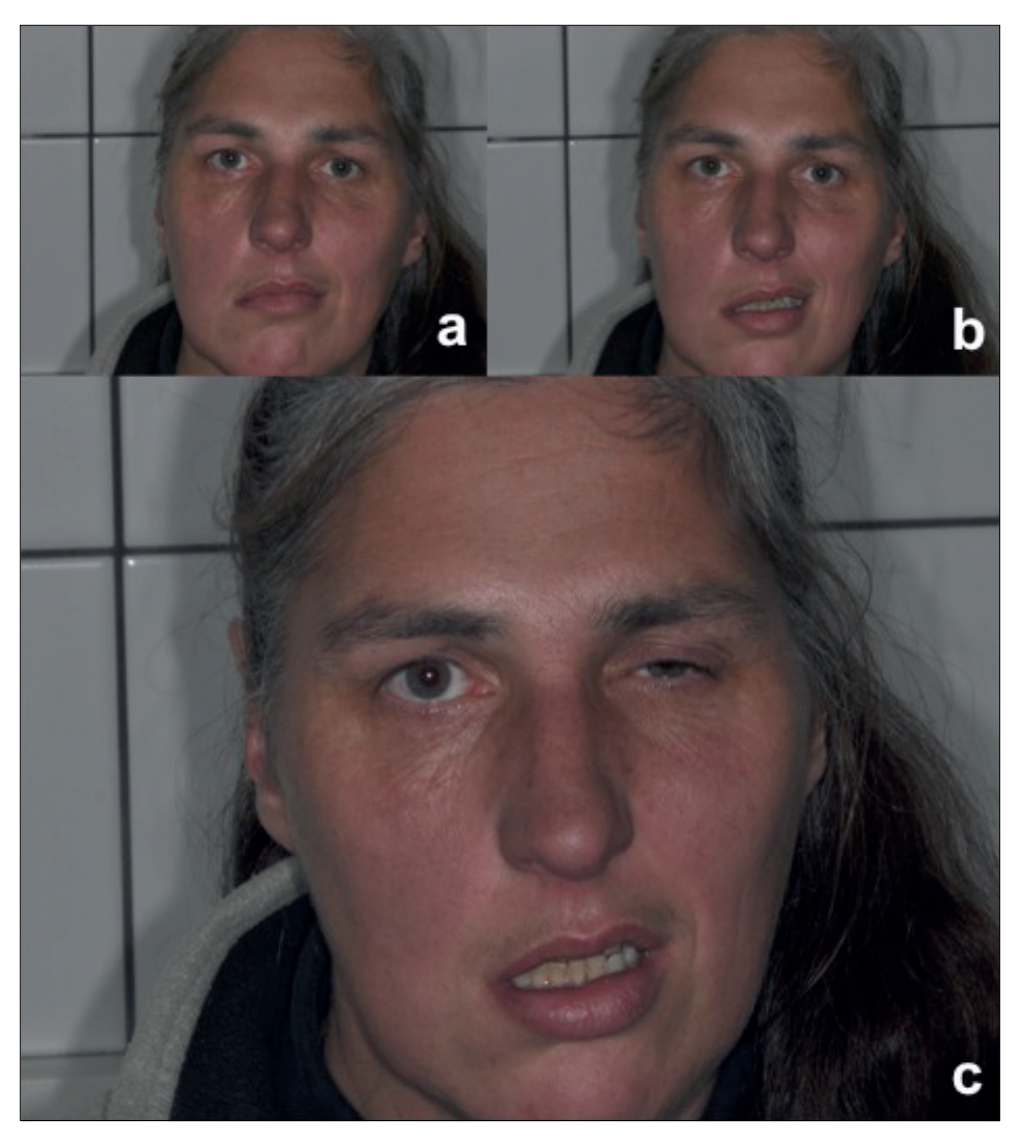

Fig. 2. Contrôle après 10 jours de traitement par aciclovir. $a$ : patiente au repos sans mimique faciale ; $b$ : patiente souriante, la lèvre droite est moins étirée ; $c$ : patiente en occlusion palpébrale, disparition du signe de Charles Bell.

Fig. 2. Control after 10 days of treatment of aciclovir. a: patient at rest without facial expression; $b$ : patient smiling, right lip is less stretched; c: patient in eyelid occlusion, disappearance of Charles Bell's sign.

varicelle dans son enfance. Les autres étiologies de la paralysie faciale (congénitale, tumorale, traumatique ou neuropathique) ont été éliminées une par une; les sérologies demandées se sont toutes révélées négatives (HSV 1, HSV 2, hépatite $B$ et $C$, Borrelia burgdorferi).

On a prescrit un collyre, des larmes artificielles et des « strips » pour maintenir la paupière en occlusion durant la nuit. Un antiviral (aciclovir, $400 \mathrm{mg} 5 \times / \mathrm{j}$ ) a été administré per-os pendant $10 \mathrm{j}$. À ce stade, la corticothérapie ne présentait plus d'intérêt.

Après $10 \mathrm{j}$ de traitement, on observait des signes d'évolution favorable : disparition du signe de Charles Bell, asymétrie du visage légèrement moins marquée : l'hypotonicité génienne droite avait diminué et la commissure labiale droite était moins étirée (Fig. 2a, b et c).

\section{Discussion}

D'autres cas similaires de paralysie faciale faisant suite à des soins dentaires ont été rapportés. Une paralysie faciale de grade IV selon House-Brackmann a été observée chez une patiente de 21 ans [2], après l'avulsion simple d'une dent de sagesse mandibulaire ; la paralysie a régressé en 3 mois après prescription de cytidine et d'uridine (famille des nucléosides Nucle $\left.0^{\circledR}\right)$. Une paralysie de grade II est survenue chez un patient de 38 ans après la dépose d'un amalgame sur une deuxième molaire mandibulaire ; une de grade IV chez un patient de 29 ans, après avulsion d'une dent de sagesse maxillaire [3] ; toutes deux ont évolué favorablement en quelques mois après prescription de corticoïdes per os.

Différents mécanismes ont été proposés pour expliquer une lésion nerveuse après un acte dentaire [4]. Le traumatisme direct apparaît la cause la plus évidente, par section iatrogène ou compression œdémateuse. L'anesthésique injecté peut être cytotoxique, la bupivacaïne et l'articaïne étant moins toxiques que la procaïne ou la tétracaïne. Il a également été suggéré que le stress d'un traitement dentaire pourrait favoriser une réactivation virale. HSV et VZV ont été incriminés, l'origine herpétique pure se différenciant par l'absence de douleur et d'érythème dans la zone de Ramsay-Hunt qui caractérisent 
l'origine zostérienne. Les cas de paralysie faciale périphérique après une anesthésie pour des soins dentaires sont très peu nombreux : 20 à 30 cas pour $100000[5,6]$. Il n'y a aucune hypothèse satisfaisante pour expliquer cette complication rare.

Pour le traitement, les données sont contradictoires et il existe différentes approches [5, 7-10]. Pour certains auteurs, la prise de corticoïdes augmente significativement la probabilité de guérison complète, l'efficacité des antiviraux serait plus aléatoire ; pour d'autres, c'est l'inverse. Les corticoïdes augmenteraient la probabilité de récupération totale et assureraient un meilleur résultat à 6 mois $[10,11]$. Ils seraient également plus efficaces chez les personnes de plus 40 ans [8]. La posologie recommandée est de $60 \mathrm{mg} / \mathrm{j}$ de prednisolone pendant $5 \mathrm{j}$, puis elle est diminuée de $10 \mathrm{mg} / \mathrm{j}$ [5]. L'efficacité des antiviraux serait plus aléatoire [4, 10, 12]. Seuls, ils seraient moins efficaces que les corticoïdes, mais ils potentialiseraient l'effet de la prednisolone lorsqu'ils sont associés. L'American Association of Neurology recommande un traitement combinant prednisolone $(1 \mathrm{mg} / \mathrm{kg} / \mathrm{j})$ et aciclovir (400 mg $5 \mathrm{x} / \mathrm{j}$ ) pendant $10 \mathrm{j}$ [9]. Tous les auteurs s'accordent pour dire qu'il faut commencer le traitement le plus tôt possible $[7,8]$; il doit être instauré dans les 72 heures après l'apparition de la paralysie, sinon ses effets diminuent.

Le traitement chirurgical n'est envisagé qu'en absence totale de récupération. Il consiste à réaliser, selon l'étiologie, une décompression chirurgicale au niveau du canal de Fallope notamment, une suture ou une greffe nerveuse.

\section{Conclusion}

La paralysie faciale périphérique est une pathologie souvent idiopathique, contraignante de par ses conséquences esthétiques et fonctionnelles, ainsi que par l'imprévisibilité de sa survenue. Aucun lien n'a été formellement établi entre paralysie faciale périphérique et soins dentaires. Fort heureusement, c'est une complication rare, avec une guérison ad integrum dans $75 \%$ des cas dans un délai de 1 à 2 mois [11].
Conflits d'intérêt : aucun

\section{Références}

1. Taillia H, de Greslan T, Renard JL, Flocard F. Examen neurologique facial à l'usage de l'odontologiste et du chirurgien maxillofacial. Implantodontie 2005;14:90-105.

2. Smith $M H$, Lung KE. Nerve injuries after dental injection: a review of the literature. J Can Dent Assoc 2006;72:559-64.

3. Vasconcelos BC, Bessa-Nogueira RV, Maurette PE, Carneiro SC. Facial nerve paralysis after impacted lower third molar surgery: a literature review and case report. Med Oral Patol Oral Cir Bucal 2006;11:E175-8.

4. Lockhart P, Daly F, Pikethly M, Comerford N, Sullivan F. Antiviral treatment for Bell's palsy (idiopathic facial paralysis). Cochrane Database Syst Re 2009;4:CD001869.

5. Murthy JMK, Saxena AB. Bell's palsy: treatment guidelines. Ann Indian Acad Neurol 2011;14:S70-2.

6. Chevalier V, Arbab-Chirani R, Tea SH, Roux M. Facial palsy after inferior alveolar block: case report and review of literature. Int J Oral Maxillofac Surg 2010;39:1139-42.

7. Gronseth GS, Paduga R. Evidence-based guideline update: steroids and antivirals for Bell palsy. Report of the guideline development subcommittee of the American Academy of Neurology. Neurology 2012;79:2209-13.

8. Christensen KF, Ovesen T, Andreassen CS. No effect of corticosteroid treatment for idiopathic facial paralysis. Dan Med J 2012;59:A4416.

9. Tzempos FH, Cocos A, Kleftogiannis M, Zarakas M, Iatrou I. Transient delayed facial nerve palsy after inferior alveolar block anesthesia. Anesth Prog 2012;59:22-7.

10. Quant EC, Jeste SS, Muni RH, Cape AV, Bhussar MK, Peleg AY. The benefit of steroids versus steroids plus antivirals for treatment of Bell's palsy: a meta-analysis. BMJ 2009;339:b3354.

11. Monini S, Lazzarino AI, Iacolucci C, Buffoni A, Barbara M. Epidemiology of Bell's palsy in Italian health district: incidence and case-control study. Acta Otorhinolaryngol Ital 2010;30:198.

12. Worster A, Keim SM, Sahsi R, Panciolo AM. Do either corticosteroids or antiviral agents reduce the risk of long-term facial paresis in patients with new-onset Bell's palsy? J Emerg Med 2010;38:518-23.

\section{Annexes}

Grade I : normal

Grade II : déficit discret, occlusion palpébrale complète sans efforts

Grade III : face symétrique au repos, occlusion palpébrale complète possible avec effort, déficit de l'élévation des sourcils, sourire asymétrique, syncinésies et hypertonies notables

Grade IV : face à peu près symétrique, occlusion palpébrale complète impossible, mouvements très asymétriques, syncinésies et hypertonies sévères

Grade V : face asymétrique au repos et hypotonique, quelques ébauches de mouvements

Grade VI : face asymétrique au repos et hypotonique, aucun mouvement

Annexe 1 : Classification de House-Brackmann [2]. 